\title{
Targeting aberrant transcriptional repression in leukemia: a therapeutic reality?
}

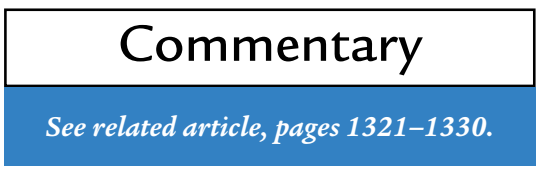

\author{
Jonathan D. Licht
}

Derald H. Ruttenberg Cancer Center and Department of Medicine, Mount Sinai School of Medicine, Box 1130, One Gustave L. Levy Place, New York, New York 10029, USA. Phone: (212) 659-5487; Fax: (212) 849-2523; E-mail: jonathan.licht@mssm.edu.

J. Clin. Invest. 108:1277-1278 (2001). DOI:10.1172/JCI200114343.

The discovery that transcription factors are frequently disrupted in leukemia has led to efforts to target these proteins with drugs. These efforts have met with limited success, partly because it has proved difficult to design and deliver into the nucleus small molecules that might break up interactions between transcription factors and their target genes or cofactors. Hence the belief that transcription factors are not "druggable." However, the pathogenic fusion proteins of leukemia have a common ability to aberrantly repress target genes, through recruitment of histone deacetylases (HDACs) and other enzymes that can be targeted by small organic molecules. Suberoylanilide hydroxamic acid (SAHA) is an HDAC inhibitor (HDI) entering clinical trials that may reverse aberrant repression by fusion proteins.

Acute promyelocytic leukemia (APL) results from a chromosomal translocation in which the retinoic acid receptor gene (RAR $\alpha$ ) becomes fused to any of five partners (1). In the absence of ligand, corepressors like $\mathrm{N}-\mathrm{CoR}$ bind to RAR, recruiting HDACs. Physiological levels of retinoic acid (RA) induce the release of corepressors and binding of coactivators with histone acetyl transferase activity. While deacetylated histone tails hinder transcription, lysine acetylation of the tails favors gene expression (2). In $t(15 ; 17)$ APL, the PML-RAR fusion protein has abnormally high affinity for corepressors and the protein switches to an activator only at pharmacological doses of RA (3). Such treatment provides one example of successful transcription-based therapy.

$\mathrm{t}(11 ; 17)$ yields the PLZF-RAR fusion protein and an RA-resistant form of the disease (1). PLZF is a transcriptional repressor (4) and complexes with corepressors and HDACs (5). Unlike

PML-RAR, the PLZF-RAR fusion protein fails to release corepressors under the influence of RA, explaining the ineffectiveness of this treatment. However, HDIs such as trichostatin A and butyrate (3) block repression of reporter genes by PLZF-RAR. Whether reversal of transcriptional repression explains the efficacy of the drug combination in vivo is unclear.

In the current issue of the JCI, He et al. (6) show that only the combination of RA and SAHA is sufficient to clear leukemic blasts from the peripheral blood of mice harboring the fusion genes of $t(11 ; 17)$ APL. This is an impor-

While transcription factors

are generally thought not

to be "druggable", the ability

of the pathogenic fusion

proteins of leukemia to recruit

histone deacetylases can

indeed be targeted by small organic molecules.

tant result that motivates potential clinical trials of the combination. However, it is not clear that SAHA acts in this system solely by reversing the effects of PLZF-RAR, since SAHA also potently affects leukemia cell apoptosis. In NB4 cells derived from a $t(15 ; 17)$ APL patient and in blasts from $t(11 ; 17)$ animals, SAHA induced apoptosis and cell cycle arrest while RA induced growth arrest accompanied by differentiation. In NB4 cells, the combination increased apoptosis, suggesting that RA, by altering gene expression, can sensitize cells to the cytotoxic effects of SAHA. SAHA itself slightly increased differentiation in NB4 cells, while in $t(11 ; 17)$ blasts, both SAHA and RA induced differentiation, with the combination yielding an additive effect. This last result can be explained equally well by overlapping independent effects or an effect of HDIs on APL fusion proteins. Another study of human $\mathrm{t}(11 ; 17)$ APL blasts found that $1 \mu \mathrm{M}$ RA alone did not induce any differentiation, while trichostatin A + RA did, offering more support for the idea that HDIs can reverse the effects of APL fusions (7).

In a microarray experiment in NB4 cells, SAHA induced a set of genes different from those induced by RA, correlating with their distinct cellular effects. SAHA leads to hyperacetylation of the p21 gene (8), but whether activation of this or other genes by the HDI activity of SAHA causes apoptosis is unknown. It should be noted that expression of p21 itself induces myeloid differentiation (9). SAHA could also alter acetylation of nonhistone proteins or, like its predecessor HMBA, affect signaling pathways to induce apoptosis. SAHA boosted expression of a few RAR target genes, and a very small number of genes were induced only in the presence of the two compounds. Again, these data support the notion that the agents work together by nonoverlapping mechanisms, not necessarily by blocking the APL fusion protein. SAHA treatment increased bulk histone acetylation in mice, but whether this caused changes in chromatin configuration around C/EBPE and other RAR target genes that are repressed by APL fusion proteins (1) was not determined. Chromatin immunoprecipitation experiments using the cells of mice before and after treatment witha combination of HDI, and RA will be a gold standard test for the transcription therapy hypothesis. 
How then can the transcription effects of the fusion proteins of APL and other forms of leukemia be specifically targeted? The answer may lie in the structure of the histone tails associated with RAR target genes. It is clear that there is a "code" of histone tail modification, including methylation, phosphorylation, and ubiquitination (10). Modified tails are contact sites for activator or repressor complexes. For example, retinoblastoma protein $(\mathrm{Rb})$ recruits histone HDACs as well as histone methyl-transferase to target genes (11). The result is the replacement of acetyl groups on histone near E2F binding sites with methyl groups, offering a new binding site for heterochromatin protein 1 (HP1) (12). HP1 can also be recruited by transcriptional repressors, altering chromatin to an inactive state. If the RAR fusion proteins also yield aberrant histone methylation at targets, HDIs would be insufficient for the formation of acetylated, active chromatin. Transcriptional repressor complexes can also be associated with DNA methyl-transferase activity (13). If APL fusion proteins recruit such enzymes, target genes might be silenced. In this case, addition of a DNA methyl-transferase inhibitor like 5 -azacytidine could be useful.

In summary, the notion of transcription-targeted therapy is highly attractive and well founded by in vitro findings. The current study shows that combinations of agents that can alter gene regulation may be of therapeutic benefit, but whether the APL fusion protein was the common target is uncertain. Nevertheless, the quest for such therapy will continue in order to find nontoxic alternatives to chemotherapy. Mechanistic and empiric trials may lead to the development of regimens of enzyme inhibitors potentially tailored to the transcriptional pathology caused by the fusion proteins of leukemia.

1. Melnick, A., and Licht, J.D. 1999. Deconstructing a disease: RARalpha, its fusion partners, and their roles in the pathogenesis of acute promyelocytic leukemia. Blood. 93:3167-3215.

2. Lee, T.I., and Young, R.A. 2000. Transcription of eukaryotic protein-coding genes. Annu. Rev. Genet. 34:77-137.

3. Lin, R.J., et al. 1998. Role of the histone deacetylase complex in acute promyelocytic leukaemia. Nature. 391:811-814.

4. Li, J.Y., et al. 1997. Sequence-specific DNA binding and transcriptional regulation by the promye- locytic leukemia zinc finger protein. J. Biol. Chem. 272:22447-22455

5.Hong, S.H., David, G., Wong, C.W., Dejean, A. and Privalsky, M.L. 1997. SMRT corepressor interacts with PLZF and with the PML-retinoic acid receptor alpha (RARalpha) and PLZF-RAR alpha oncoproteins associated with acute promyelocytic leukemia. Proc. Natl. Acad. Sci. USA. 94:9028-9033

6. He, L.-Z., et al. 2001. Histone deacetylase inhibitors induce remission in transgenic models of therapy-resistant acute promyelocytic leukemia. J. Clin. Invest. 108:1321-1330.

7. Kitamura, K., et al. 2000. Histone deacetylase inhibitor but not arsenic trioxide differentiates acute promyelocytic leukaemia cells with $\mathrm{t}(11 ; 17)$ in combination with all-trans retinoic acid. $\mathrm{Br} . \mathrm{J}$. Haematol. 108:696-702.

8. Richon, V.M., Sandhoff, T.W., Rifkind, R.A., and Marks, P.A. 2000. Histone deacetylase inhibitor selectively induces p21WAF1 expression and gene-associated histone acetylation. Proc. Natl. Acad. Sci. USA. 97:10014-10019.

9. Liu, M., Lee, M.H., Cohen, M., Bommakanti, M., and Freedman, L.P. 1996. Transcriptional activation of the Cdk inhibitor $\mathrm{p} 21$ by vitamin $\mathrm{D} 3$ leads to the induced differentiation of the myelomonocytic cell line U937. Genes Dev. 10:142-153.

10. Jenuwein, T., and Allis, C.D. 2001. Translating the histone code. Science. 293:1074-1080.

11. Nielsen, S.J., et al. 2001. Rb targets histone H3 methylation and HP1 to promoters. Nature. 412:561-565.

12. Bannister, A.J., et al. 2001. Selective recognition of methylated lysine 9 on histone $\mathrm{H} 3$ by the HP1 chromo domain. Nature. 410:120-124.

13. Rountree, M.R., Bachman, K.E., and Baylin, S.B. 2000. DNMT1 binds HDAC2 and a new corepressor, DMAP1, to form a complex at replication foci. Nat. Genet. 25:269-277. 